\title{
Comparison of Student Performance When Solving a THERMOdYNAMIC CYCLE after Implementing an Alternative Teaching Method
}

\author{
Juan Abellól and Douglas Ruth ${ }^{2}$ \\ ${ }^{1}$ University of British Columbia, ${ }^{2}$ University of Manitoba \\ abello@mech.ubc.ca,douglas.ruth@umanitoba.ca
}

\begin{abstract}
First-year engineering students at the University of Manitoba take a thermodynamics course. The summer instructor taught the course differently from the fall and winter terms. He combined tutorials with lectures to introduce active learning to the course, implemented an online problem library and increased the number of term tests.

Students in the summer 2016 term were given a similar thermodynamic cycle question in their final exam as students in the winter 2016 term. Student performance in the cycle question was compared in order to evaluate the effectiveness of the new teaching method.

Both groups had similar question averages with generally similar question score histograms. However, winter students scored an average of $10 \%$ below their GPA, while summer students scored an average of $1 \%$ below their GPA. The difference between these averages is statistically significant $(97.5 \%$ confidence). These results suggest that the new teaching approach leads to better student performance when solving thermodynamic cycles.
\end{abstract}

Keywords: Student performance, active learning, thermodynamics, thermodynamic cycle.

\section{INTRODUCTION}

Introduction to Thermal Sciences (ENG 1460) is a first-year thermodynamics course at the University of Manitoba. It covers simple hydrostatics, properties of a pure substance, first law applied to closed and open systems, and second law. The course is required for all engineering students. It counts for four credit hours.

The course runs two parallel sections in the fall term, three in the winter term and one in the summer term. Each section has a maximum enrollment of 80 students. The fall and winter term sections are taught in a traditional format consisting of three 50-minute lectures or two 75-minute lectures per week, plus one 110-minute tutorial per week. The summer section is scheduled for four sessions per week, with two of these sessions lasting 140 minutes each and the other two lasting 110 minutes each. There are typically 5-6 instructors teaching the course in any given academic year.

The longer scheduled times in the summer allowed the instructor to practice a format that combined lecture with tutorial. In each session, the instructor started by demonstrating a sample problem. Then he polled students using clickers to gage student understanding of the solution process. If most students understood the sample problem, the instructor gave students a tutorial problem of similar scope and typically higher difficulty. Students solved this problem in class while discussing with their peers. (The summer course is held in a classroom with round tables that accommodate 4-6 students each.) The instructor and teaching assistants circulated through the room to answer any questions. Once sufficient time had passed, the instructor reviewed the problem solution and moved onto the next example or topic. Students collected the problems they solved during the weekly sessions, and submitted them as part of their homework at the end of the week. This approach is discussed in more detail in references 1 and 2. Student feedback collected during the summer term appears in reference 2.

The summer instructor also introduced two additional changes to the course. First, he had students write an additional term test that covered first law applied to open systems. This motivated students to prepare this material (and allowed them to receive feedback) before writing the final exam. Second, he led the development of a library of online quizzes and practice problems that were available to students through the university learning management system. These practice problems were structured in a way that guided students through the solution process, provided hints if needed and gave immediate feedback after completing a problem. Further details on the development of this online tool appear in reference 3.

Preliminary results showed that students who completed ENG 1460 during the summer term earned a course grade that was generally higher than their GPA [2]. 
The course instructors wished to know how this compared to students taking the course during the fall or winter sessions. One way to make this comparison was to give summer and winter students the same (unreleased) problem on a final exam. This paper presents the results of this investigation.

\section{METHODOLOGY}

Final exams for ENG 1460 include a question where students analyze a thermodynamic cycle consisting of several open-flow devices. Solving this question requires knowledge of how to determine properties of a pure substance, how to apply the first law to an open system and how to apply the second law to a cycle. This question is usually worth $25-35 \%$ of the total exam marks. Students have access to some previous final exams, and know to expect one question of this type.

The winter 2016 final exam for ENG 1460 was not released. All students who wrote that exam returned the questions sheets and their solutions. That kept the questions unknown to the summer 2016 students. The summer 2016 final exam contained the same thermodynamic cycle question as the winter 2016 final exam.

A metric to compare student performance was created as follows. The cumulative GPA for each student up to the term preceding the exam was used to predict the score of each student in the thermodynamic cycle question. (This assumed that each student would perform at a level equivalent to his or her GPA.) Then the difference between the actual and predicted question score (DQS) was calculated. The average of this difference was then calculated for the winter 2016 students and compared to that of the summer 2016 students.

For example, suppose three students solved a thermodynamic cycle question in their winter 2016 final exam (table 1). The fall 2015 GPA of these students can be used to calculate their predicted cycle question score. The difference between the actual and predicted cycle question score DQS is found and averaged. In this example, the winter 2016 students scored an average of $8 \%$ below their GPA. (The example data shown in table 1 is for illustration purposes. Sample calculations leading to this table are shown in appendix A.1.)

Table 1: Example winter 2016 student data.

\begin{tabular}{|c|c|c|c|c|}
\hline Student & $\begin{array}{c}\text { Fall 2015 } \\
\text { GPA }\end{array}$ & $\begin{array}{c}\text { Predicted } \\
\text { score }(\%)\end{array}$ & $\begin{array}{c}\text { Actual } \\
\text { score }(\%)\end{array}$ & $\begin{array}{c}\text { DQS } \\
(\%)\end{array}$ \\
\hline W1 & 4.0 & 89 & 72 & -17 \\
\hline W2 & 3.0 & 67 & 70 & 3 \\
\hline W3 & 3.5 & 78 & 68 & -10 \\
\hline Average & & & & -8 \\
\hline
\end{tabular}

Now suppose that two students solved the same thermodynamic cycle question in their summer 2016 final exam (table 2). The same procedure previously outlined can be used to determine that students in this group scored an average of $1 \%$ above their GPA. (The example data shown in table 2 is for illustration purposes. Sample calculations leading to this table are shown in appendix A.2.)

Table 2: Example summer 2016 student data.

\begin{tabular}{|c|c|c|c|c|}
\hline Student & $\begin{array}{c}\text { Winter } \\
\text { 2016 GPA }\end{array}$ & $\begin{array}{c}\text { Predicted } \\
\text { score (\%) }\end{array}$ & $\begin{array}{c}\text { Actual } \\
\text { score (\%) }\end{array}$ & $\begin{array}{c}\text { DQS } \\
(\%)\end{array}$ \\
\hline S1 & 4.0 & 89 & 82 & -7 \\
\hline S2 & 3.0 & 67 & 76 & 9 \\
\hline Average & & & & 1 \\
\hline
\end{tabular}

The results of this example can now be compared. The winter 2016 example students scored an average of $8 \%$ below their GPA while the summer 2016 example students scored $1 \%$ above their GPA. Statistical analysis can then be performed to determine whether this difference is statistically significant.

Our findings when applying the analysis methods described above to the actual cycle question scores appear in the following section.

\section{RESULTS}

We analyzed data for 39 students in the winter 2016 term and 68 students in the summer 2016 term. (Students for whom we had no GPA data for the previous term could not be counted in this study.) The winter 2016 students averaged $69 \%$ in the cycle question. The summer 2015 students obtained the same average. A histogram showing the cycle question score distribution for the winter 2016 and summer 2016 students appears in figure 1 . These results show that the question averages and distributions are similar for both groups.

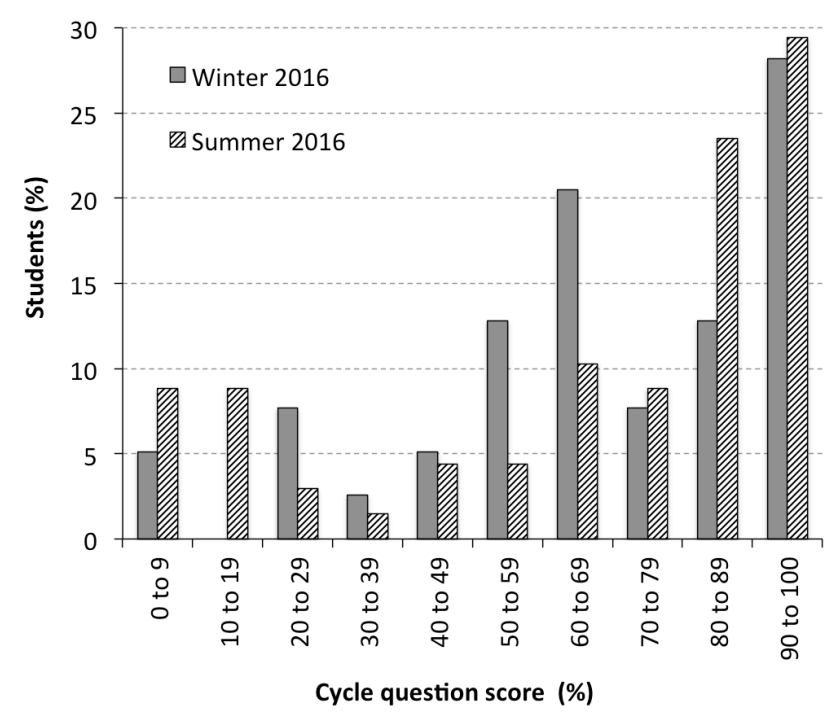

Figure 1: Histogram of the cycle question score. 
Even though the average and the histogram of the cycle question scores were similar for both student groups, the average difference between actual and predicted scores DQS was $-10 \%$ for the winter 2016 students and $-1 \%$ for the summer 2016 students. This indicates that both groups performed below their GPA, but summer 2016 students performed noticeable closer to their GPA than winter students. The difference between average DQS for these groups (-10\% vs. $-1 \%)$ was found to be statistically significant with confidence of $97.5 \%$.

A histogram of the difference between actual and predicted cycle question scores DQS appears in figure 2. The DQS distribution is roughly similar for winter 2016 and summer 2016 students, but the summer 2016 distribution is shifted to the right. This corresponds to the summer 2016 students having a smaller negative DQS $(-1 \%)$ than the winter 2016 students $(-10 \%)$.

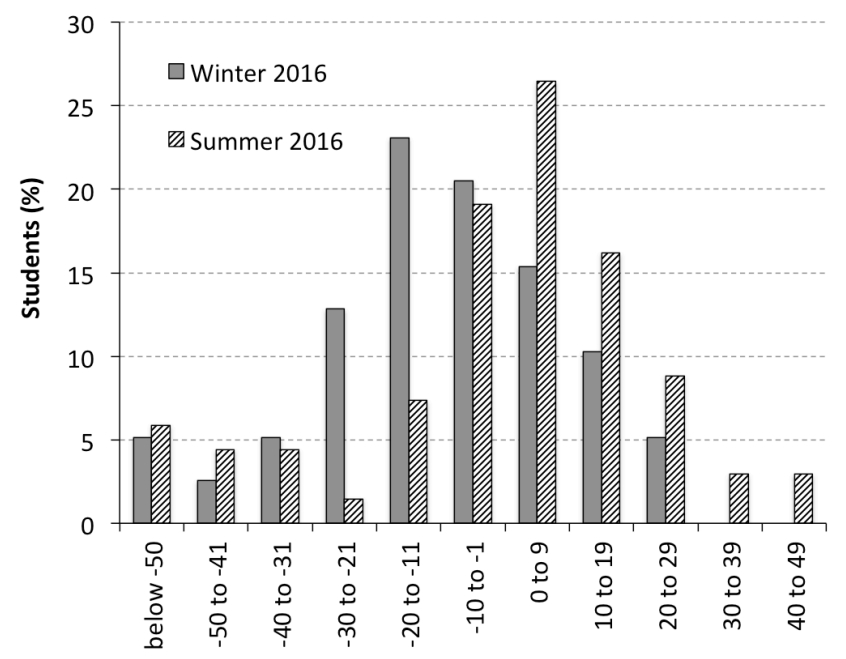

[Actual] - [predicted] cycle question score (\%)

Figure 2: Histogram of the difference between actual and predicted cycle question score DQS.

Further observations can be made after separating students in GPA groups and comparing the average difference between actual and predicted cycle question scores DQS for each GPA group. B students scored at their GPA level in the summer 2016 term but averaged $11 \%$ below their GPA for the winter 2016 term. This difference in average DQS was found to be statistically significant with confidence of $95 \%$. Preliminary results indicate $\mathrm{C}$ students also scored below but closer to their GPA in the summer 2016 term, while A students performed at similar DQS in both terms. Our data set does not have enough data to analyze D students.

\section{CONCLUSIONS}

A teaching approach that combines tutorial with traditional lectures, introduces an additional term test consisting of thermodynamic cycles and uses a library of online questions improves student performance (relative to student GPA) in thermodynamic cycle problems. Students in the B range benefit from this approach. Preliminary results indicate that $\mathrm{C}$ students also benefit, while A students do equally well in the alternate or the traditional teaching approaches.

Additional factors that the authors recognize as helpful when teaching thermodynamic cycles include fixing all relevant states prior to analyzing the components in the cycle, emphasizing a systematic approach that uses the same steps to simplify the general first-law equation when applying it to different components, and teaching about open-flow devices in the context of a larger cycle.

\section{References}

[1] Jillian Seniuk Cicek, Douglas Ruth and Sandra Ingram, "Thermodynamics is not a spectator sport!": an exploratory study on incorporating active learning into a first year thermodynamics course" in Proc. CEEA Canadian Engineering Education Conf., Montreal, QC; 1820 June 2013.

[2] Jillian Seniuk Cicek, Douglas Ruth and Sandra Ingram, "Redesigning the learning experience: one professor's efforts to develop an active and engaging first-year thermodynamics course" in Proc. CEEA Canadian Engineering Education Conf., Canmore, AB; 8-11 June 2014.

[3] Joyce Valencerina, Douglas Ruth and Jillian Seniuk Cicek, "Design of mastery-based tutorial in the Brightspace learning environment for a first-year thermodynamics course" in Proc. CEEA Canadian Engineering Education Conf., Halifax, NS; 19-22 June 2014.

\section{APPENDIX: SAMPLE CALCULATIONS}

This appendix presents the calculations behind example tables 1 and 2. These data are for illustration purposes only.

\section{A.1 Table 1}

Student W1 has a GPA of 4.0/4.5 To score at this GPA level, the cycle question score should be $4.0(100 \% / 4.5)=89 \%$. The actual score for this student is $72 \%$, so the difference between actual and predicted question score is DQS $=72 \%-89 \%=-17 \%$.

Student W2 has a GPA of 3.0/4.5 To score at this GPA level, the cycle question score should be $3.0(100 \% / 4.5)=67 \%$. The actual score for this student 
is $70 \%$, so the difference between actual and predicted question score is DQS $=70 \%-67 \%=3 \%$.

Student W3 has a GPA of 3.5/4.5 To score at this GPA level, the cycle question score should be $3.5(100 \% / 4.5)=78 \%$. The actual score for this student is $68 \%$, so the difference between actual and predicted question score is DQS $=68 \%-78 \%=-10 \%$.

The average DQS for the winter students W1, W2 and $\mathrm{W} 3$ is $(-17 \%+3 \%-10 \%) / 3=-8 \%$.

\section{A.2 Table 2}

Student S1 has a GPA of 4.0/4.5 To score at this GPA level, the cycle question score should be
$4.0(100 \% / 4.5)=89 \%$. The actual score for this student is $82 \%$, so the difference between actual and predicted question score is DQS $=82 \%-89 \%=-7 \%$.

Student S2 has a GPA of 3.0/4.5 To score at this GPA level, the cycle question score should be $3.0(100 \% / 4.5)=67 \%$. The actual score for this student is $76 \%$, so the difference between actual and predicted question score is DQS $=76 \%-67 \%=9 \%$.

The average DQS for the summer students S1 and S2 is $(-7 \%+9 \%) / 2=1 \%$. 